\title{
Dynamical TEM Investigation of Solidification Kinetics in AZ91 Mg Alloys
}

\author{
Karen Kruska ${ }^{1}$, Aashish Rohatgi ${ }^{2}$, Rama Shesa Vemuri ${ }^{3}$, James E Evans ${ }^{1}$, Libor Kovarik ${ }^{3}$, Patricia \\ Abellan Baeza ${ }^{1}$, Lucas R Parent ${ }^{1}$, Layla Mehdi ${ }^{1}$ and Nigel DBrowning ${ }^{1}$ \\ ${ }^{1}$ Fundamental and Computational Science Directorate, Pacific Northwest National Laboratory, \\ Richland, WA, USA \\ ${ }^{2}$ Energy and Environment Directorate, Pacific Northwest National Laboratory, Richland, WA, USA \\ ${ }^{3}$ Environmental Molecular Sciences Laboratory, Pacific Northwest National Laboratory, \\ Richland, WA, USA
}

The recent developments in climate and environmental politics have made the quest for new lightweight materials that will decrease a cars overall weight and fuel consumption a focus of interest for the automotive industry. In this regard, magnesium alloys have a high strength-to-weight ratio and are therefore a great candidate material to replace structural Al alloys and steels to reduce component weight. Al-containing $\mathrm{Mg}$ alloys have good mechanical strength and corrosion resistance at room temperature (RT), but these properties deteriorate at the elevated temperatures $\left(60-200^{\circ} \mathrm{C}\right)$ that automotive components are commonly exposed to [1]. Understanding the microstructural evolution in this system is therefore key to the use of these lightweight alloys in the future.

Favorable properties in $\mathrm{Mg}$ alloys can be achieved by high pressure die-casting. High cooling rates lead to the formation of a non-equilibrium microstructure comprising $\mathrm{Mg}_{17} \mathrm{Al}_{12}$ precipitates $(\beta)$ and $\mathrm{Mg}-\mathrm{Al}$ solid-solution $(\alpha)$ [2]. Minor alloying elements, such as $\mathrm{Zn}$ or Fe, are sometimes added to further improve the alloy properties. While it is widely accepted that the casting thickness [3], which directly influences the local cooling rate, is a crucial factor in the resulting alloy properties, the kinetics driving the solidification and formation of the $\alpha$-phase are not well understood. As the cooling rates during high pressure die-casting are high $(\sim 100-1000 \mathrm{~K} / \mathrm{s})$ the solidification kinetics and their implications for phase formation and stability are difficult to access. The recording speed in conventional TEM is usually limited by the refresh rate of the CCD (or other detector) and insufficient to investigate high-rate solidification kinetics. Therefore, this study uses a novel dynamical TEM (DTEM) approach to capture the phase formation in real time. Rather than imaging with a continuous electron beam as in a conventional TEM, a dynamical TEM uses a pulsed laser to generate discrete electron bursts that can capture ultrafast reactions [4]. A schematic of the geometry of the experiment is shown in Figure 1. Insitu laser melting was employed to simulate the ultrafast cooling rates during the solidification process in a TEM foil and the laser induced electron pulses with a well-defined delay were used to capture the solidification process in real-time.

Thin nanocrystalline AZ91 films, up to $100 \mathrm{~nm}$ thick, were sputter-coated on a TEM grid. The C-film was subsequently removed leaving the pristine metal film supported by the $\mathrm{Cu}$-grid. This geometry allows the most flexibility for performing multiple experiments on the same sample during a DTEM experiment. This is often necessary to improve the count rates and make it possible to interpret the images. Imaging and local area diffraction (LAD) can be used to observe the microstructure evolution during melting and solidification with the resulting diffraction patterns being compared to continuous beam diffraction patterns and simulated patterns. The volume fraction of solid and liquid phases, growth rates of dendrites and nucleation and growth of $\mathrm{Mg}_{17} \mathrm{Al}_{12}$ precipitates can then be determined as a 
function of time. Although interpretation of micrographs was not always straight forward, the nonequilibrium $\alpha-\mathrm{Mg}$ phase can typically be identified.

Another advantage in using the dynamical TEM approach to study the non-equilibrium phase formation during solidification of low-melting point metals in real-time is that liquid and solid phases can be identified with high spatial and temporal resolution. Such information can be used to guide deterministic micromodeling in the commercial casting package ProCAST with the help of back diffusion thermodynamic calculations and its thermal-physical properties prediction capabilities [5].

[1] G L Song, A L Bowles, and D H StJohn, Materials Science and Engineering a-Structural Materials Properties Microstructure and Processing 366 (2004), p.74-86.

[2] A K Dahle, Y C Lee, M D Nave, P L Schaffer and D H StJohn, Journal of Light Metals 1 (2001) p. $61-72$.

[3] C H Cáceres, J W Poole, A L Bowles and C J Davidson, Materials Science and Engineering A 402 (2005) p. $269-277$.

[4] A Kulovits, J M K Wiezorek, T LaGrange, B W Reed and G H Campbell, Philosophical Magazine Letters 91 p. $287-296$.

[5] This work was sponsored by Will Joost, program manager for the Vehicle Technologies Office of the U.S. Department of Energy. The development of the DTEM capability is part of the Chemical Imaging Initiative at Pacific Northwest National Laboratory under Contract DE-AC05-76RL01830 operated for DOE by Battelle. A portion of the research was performed using EMSL, a national scientific user facility sponsored by the Department of Energy's Office of Biological and Environmental Research and located at Pacific Northwest National Laboratory.

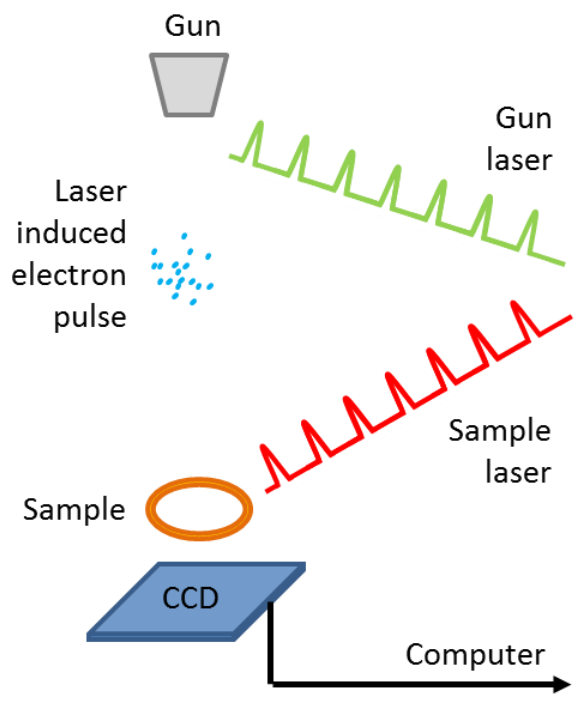

Figure 1. Schematic of a DTEM experiment. 